\title{
Emprendimiento, innovación y compromiso social en las Universidades Públicas Estatales en México
}

\section{Entrepreneurship, innovation and social commitment in Higher Education Institutions (HEI) in Mexico}

\author{
Maribel González-Cadena ${ }^{a}$; Angélica M. Vázquez-Rojas ${ }^{b}$; Myriam Coronado-Meneses ${ }^{c}$
}

\begin{abstract}
:
The purpose of this paper is to analyze the activities of entrepreneurship, innovation and social commitment in Higher Education Institutions (HEI) in Mexico, from 2007 to the present. By analyzing the literature and reviewing the database, we can distinguish the degree of progress these institutions have made in their development. The State Public Universities (UPEs) carry out the three types of activities, which has allowed them to be recognized for its work. It should be noted that entrepreneurship is the activity that predominates in the progress of these institutions towards the fulfillment of the Third Stream; however, the activities related to social commitment are the ones that present less progress compared to the other two.
\end{abstract}

Keywords: Third stream; State Public University; entrepreneurship, innovation and social commitment

\section{Resumen:}

El presente trabajo tiene como propósito analizar las actividades de emprendimiento, innovación y compromiso social en las Instituciones de Educación Superior (IES) en México a partir del año 2007 a la actualidad. Mediante el análisis de la literatura y revisión en base de datos se distingue el grado de avance que han tenido dichas instituciones en su desarrollo y se desprende que las Universidades Públicas Estatales (UPEs) efectúan los tres tipos de actividades, lo que ha permitido que éstas sean reconocidas por la labor que realizan dentro de la sociedad. Cabe destacar que la actividad de emprendimiento predomina en el avance de dichas instituciones hacia el cumplimiento de la Tercera Misión; sin embargo, las actividades referentes al compromiso social son las que presentan menor avance en comparación a las otras dos.

Palabras Clave:

Tercera Misión; Universidades Públicas Estatales; emprendimiento, innovación y compromiso social

\section{Introducción}

A finales del siglo $\mathrm{XX}$ los organismos financieros internacionales establecen una serie de recomendaciones a las instituciones educativas con el objetivo de aplicarlas en cada uno de los niveles. En este tenor, la Organización de las Naciones Unidas para la Educación, la Ciencia y la Cultura (UNESCO), en su informe final "La educación superior en el siglo XXI, visión y acción”, señala que la calidad en la educación superior es impartida por docentes actualizados que transmiten su conocimiento por medio de la docencia e investigación al estimular la innovación permanente y, por ende, necesita fuentes de financiamiento para mejorar la calidad y pertinencia en sus programas educativos (UNESCO, 1998).

En este contexto, se establece el Plan de Acción para la Transformación de la Educación Superior en América Latina y el Caribe, el mismo refiere que el conocimiento y la innovación tecnológica-productiva que se genera dentro de las Instituciones de Educación Superior (IES) ayudan a alcanzar el desarrollo económico y social de la región y, para lograr dicho fin, es necesario el financiamiento por parte del Estado y de los Organismos (UNESCO, 1998).

De igual forma, la Organización para la Cooperación y el Desarrollo Económico (OCDE, 2007) establece que las IES tienen un papel muy importante dentro del desarrollo económico, social y cultural en los países y entre sus principales recomendaciones, se encuentran:

- Mayor vinculación con pequeñas y medianas empresas;

- Ampliar su portafolio de servicios a las empresas;

- Preparar a los estudiantes para que puedan enfrentarse al mundo globalizado.

a Maribel González-Cadena, Universidad Autónoma del Estado de Hidalgo, Escuela Superior de Tizayuca, email: maribel_gonzalez4257@uaeh.edu.mx

b Angélica María Vázquez-Rojas, Universidad Autónoma del Estado de Hidalgo, Instituto de Ciencias Económico-Administrativas, email: callinalli@yahoo.com.mx

c Myriam Coronado-Meneses Universidad Autónoma del Estado de Hidalgo, Escuela Superior de Tizayuca, email myriam_coronado3187@uaeh.edu.mx 
En este tenor, las universidades, además de cumplir con su Primera (docencia) y Segunda Misión (investigación), ahora requieren ser emprendedoras e innovadoras para dar respuesta a lo señalado por los diferentes organismos internacionales; por lo tanto, Clark (1998) define a la universidad emprendedora como "universidades que maximizan el potencial de comercialización de sus ideas y crean valor en la sociedad".

Por su parte, Vialta (2013) señala que las IES buscan apoyar al desarrollo social y económico a través de una nueva misión que se fundamente en la responsabilidad social institucional de la universidad y en su compromiso de transformar el conocimiento en valor económico mediante la realización de actividades claves, tales como producir conocimiento aplicable y fomentar la innovación, formar profesionales cualificados a lo largo de la vida, fomentar proyectos de emprendedores en colaboración con el resto de agentes del sistema económico (Associació Catalana d’Universitats Públiques, 2008).

Esta nueva misión, conocida como Tercera Misión, de acuerdo con Galindo, Sanz y De Benito (2011:112) se define como:

[...] aquellas actividades que tienen relación a la generación, aplicación, uso y explotación de la tecnología y el conocimiento de la universidad fuera de la misma, mediante la interacción de la sociedad. Sin embargo, no solo se trata de generar innovación y aplicarla: sino que la Universidad ha adquirido una responsabilidad y compromiso corporativo con la sociedad a la que pertenece y con su entorno más próximo.

A partir de estas definiciones Bueno y Casiani (2007) proponen tres direcciones, con la finalidad de identificar las líneas de acción, que ayudan a cumplir la Tercera Misión de las universidades. La primera está fundamentada en el emprendimiento, como creador de valor, riqueza y empleo; la segunda, en la transferencia del conocimiento; y por último, en la extensión de sus actividades hacia el desarrollo económico y social de su comunidad.

\section{Tercera Misión en las Instituciones de Educación Superior en México}

Desde el año 2007, en México, el gobierno por medio de sus diferentes programas sectoriales de educación, recomienda a las IES que instauren estrategias y acciones que permitan realizar actividades de emprendimiento, innovación y compromiso social, con la finalidad de ayudar a impulsar el desarrollo económico y social del país, para dar respuesta a lo solicitado por los organismos internacionales.

Bajo estas condiciones, las IES han realizado diferentes actividades que les permite ir incorporando en sus planes de desarrollo, misión y visión actividades de emprendimiento, innovación y compromiso social.

En un reciente estudio de las IES mexicanas (Miranda et al., 2016), señalan que éstas se encuentran en un proceso de adaptación con la vinculación de empresas, el establecimiento de programas educativos pertinentes, el incremento o desarrollo de redes de universidades, acciones de cooperación en materia de investigación e innovación, a través de la transferencia de tecnología y conocimiento, la formación de personas calificadas que ayuden a resolver problemas reales de la sociedad mexicana.

Por otra parte, la Asociación Nacional de Universidades e Instituciones de Educación Superior (ANUIES), en su Plan de Desarrollo Institucional. Visión 2030 (ANUIES, 2016), reconoce que las instituciones educativas que pertenecen a dicha asociación han realizado acciones enfocadas con la responsabilidad social que tienen hacia la sociedad; no obstante, es necesario que exista un modelo universitario que vaya acorde a las características de estas instituciones, que les permitan alcanzar resultados socialmente significativos y que sean reconocidas en la sociedad por su participación en este tipo de actividades.

\section{Sistema Universitario Mexicano}

El Sistema de Educación Superior Pública (SESP) ofrece distintas opciones de formación con base en los intereses y objetivos profesionales que los alumnos deseen alcanzar. Dicho SESP está conformado por siete subsistemas de los cuales, solo las Universidades Públicas Federales (UPF), las Universidades Públicas Estatales (UPEs), las Universidades Públicas Estatal con Apoyo Solidario (UPEAS) y los Institutos Tecnológicos (IT) desarrollan funciones de docencia, generación y aplicación innovadora del conocimiento y cuentan con programas y proyectos de investigación, además de extensión y difusión de la cultura. El resto, no realizan actividades de investigación, desarrollo e innovación (I+D+i) o no contemplan actividades relacionadas a los indicadores que miden el compromiso social, de acuerdo a Molas-Gallart, Salter, Patel, Scott y Duran (2002).

Con base en la revisión documental se rescata lo siguiente: las UPEs son las IES que concentran el $48.29 \%$ del total de la población estudiantil nacional; así como también, el $52.08 \%$ del total de docentes y el $54.34 \%$ de los profesores investigadores que pertenecen al Sistema Nacional de Investigadores (SNI) en todo México; sin embargo, el presupuesto destinado para dichas instituciones no es el más alto, ya que se encuentran por debajo de las UPF.

Además, el $100 \%$ de las UPEs están asociadas a la ANUIES y el $85.29 \%$ al Consorcio de Universidades Mexicanas (CUMex), organismos que tienen como finalidad el aseguramiento de la calidad en los programas educativos, el desarrollo de funciones de docencia, investigación y extensión. 


\section{Planteamiento del problema}

A partir de los lineamientos instituidos por organismos internacionales, tales como la UNESCO y la OCDE, las UPEs tienen el compromiso de establecer estrategias de emprendimiento, innovación y compromiso social, para fomentar el desarrollo de su Tercera Misión.

Aunado a esto, el desarrollo de dichas actividades dentro de las IES ayuda a México a incrementar su nivel de competitividad que, de acuerdo con Índice Global de Competitividad del Foro Económico Mundial de la edición 2015-2016, ocupa el número 57 atrás de Chile (35), Panamá (50) y Costa Rica (52), países que han impulsado su innovación a través del incremento de la participación de las universidades (World Economic Forum, 2016).

En este sentido, es necesario que las IES mexicanas incrementen su participación en el desarrollo de dicha misión con la finalidad de dar respuesta a las diferentes necesidades que tiene la sociedad permitiendo el desarrollo económico del país por medio de actividades de emprendimiento, innovación y compromiso social.

Frente a esta nueva realidad de las IES, se han realizado estudios para conocer su avance en el desarrollo de actividades de la Tercera Misión; no obstante, en la mayoría se analiza el emprendimiento, ya que es relativamente fácil de medir; sin embargo, con esto no se logra cuantificar el grado de cumplimiento de cada uno de los indicadores que coadyuven a la realización de esta nueva misión.

Por lo tanto, se hace necesario que, a través de estudios donde se aborden los tres componentes de la Tercera Misión, se genere información de primera mano para los tomadores de decisiones en el ámbito educativo y mediante la consideración de las recomendaciones que se conciben en este tipo de estudio se contribuya al favorecimiento de la innovación, la cooperación social y la vinculación con el entorno para la generación de ingresos adicionales que puedan obtener las IES mexicanas, en particular las UPEs.

En virtud de lo anterior, el presente trabajo se orienta al análisis de los tres componentes en las UPEs, lo cual coadyuvará a la realización de la Tercera Misión.

\section{Objetivo General}

Identificar la situación actual de las actividades de emprendimiento, innovación y compromiso social que realizan las UPES en México con la finalidad de conocer si son significativas con la vinculación de la sociedad.

\section{Materiales y Método}

Este trabajo de investigación posee características de corte documental. La técnica para el análisis e interpretación de la información es a través del análisis de contenido de los documentos provenientes de fuentes primarias y secundarias.
Para la recolección de datos se utilizaron las siguientes bases: Anuario 2016-2017 de ANUIES, Principales cifras del Sistema Educativo Nacional 2017-2018 de la SEP, Estudio Comparativo de las Universidades Mexicanas de la Universidad Nacional Autónoma de México (2018) y Sistema Nacional de Investigación vigentes del CONACYT (2017) utilizando los indicadores de Molas-Gallart et al. (2002).

\section{Marco Teórico}

Para sustentar este trabajo de investigación se considera a la Nueva Teoría de Institucional (NEI), (Coase, 1937, Williamson, 1979, Ostrom, 1990 y North, 1993). Esta teoría se centra en que las instituciones son importantes y, por lo tanto, son susceptibles de ser analizadas utilizando un lenguaje económico.

North (1990) considera que las instituciones ayudan a disminuir la incertidumbre por medio del establecimiento de reglas, leyes, normas y contratos, debido a que proporcionan estructuras confiables y eficientes. Con relación al establecimiento de reglas en una institución Searle (2005:19) refiere que éstas auxilian a crear funciones de estatus, las cuales se realizan o se siguen por el reconocimiento o por aceptación de poder. Por lo tanto, las reglas institucionales van formando la estructura de las UPEs a través de la adopción de mitos racionalizados con el transcurso del tiempo.

Por su parte, autores como Larrán-Jorge y Andrades-Peña (2015:98) mencionan que dicho enfoque se orienta al estudio de la formación de reglas y normas que se dan dentro de las instituciones como una forma de organización para el logro de sus metas y que, a la vez, les permite adquirir cierto prestigio con relación a otras.

En este sentido, Carrasco y Castaño (2012:45) señalan que el establecimiento de las normas, reglas y estrategias ayudan a reducir la incertidumbre y surgen de manera deliberada o de forma espontánea.

Vaca Moreno y Riquel (2007: 3138) exhiben que existe un entorno institucional compuesto por normas y valores de los distintos agentes y, en función de ello, las organizaciones adoptan sus estructuras y procesos para agradarlos llegando a tomar un status de regla en las mismas organizaciones.

Los seguidores de la NEI consideran que las organizaciones se vuelven isomorfas por la influencia de los fenómenos que se desarrollan en sus contextos institucionales.

Scott (citado en Llamas et al., 2005) señala que estas presiones denominadas los tres pilares de las instituciones: estructuras reguladoras, cognitivas y normativas, ayudan a las mismas a tener estabilidad y sentido en sus actos que realizan para alcanzar sus objetivos establecidos.

El isomorfismo institucional, de acuerdo con Powell y DiMaggio (1999), es el resultado de los tres pilares de las instituciones y se produce por medio de tres tipos de 
elementos que originan un cambio institucional: la coerción, el mimetismo y la normalización.

En el caso de las UPEs el tipo de isomorfismo que se da es el de coerción, debido a que proviene de una obligación o condicionamiento $\mathrm{y}$, como ellas dependen del financiamiento público cambian o modifican sus estructuras para cumplir con lo solicitado. Sin embargo, autores como Guzmán y Trujillo (2008) señalan que cuando las IES someten a procesos de acreditación sus programas educativos, están siguiendo un isomorfismo normativo, debido a que los criterios de acreditación son establecidos por asociaciones de profesionistas que definen los requisitos mínimos de los profesionales en el campo y, que las instituciones cumplen para obtener el reconocimiento nacional o internacional, en su caso.

Asimismo, De la Torre, Casiani y Sagarra (2018), señalan que no solo el acreditamiento internacional de los programas educativos en las universidades hacen que sigan un isomorfismo normativo, estos autores agregan que la clasificación universitaria internacional y los sistemas de evaluación basados en el rendimiento han provocado que las IES europeas mejoren sus procedimientos y actividades con la finalidad de lograr posicionarse en mejores lugares de los rankings internacionales; como consecuencia para ellos, el isomorfismo normativo en las IES ha tenido mayores beneficios que perjuicios.

\section{Principales resultados}

Para responder al objetivo de investigación, se consideran los indicadores de Molas-Gallart et al (2002) que incluyen 12 categorías, donde se analizan tanto las actividades como las capacidades de las universidades; la investigación documental permitió identificar las principales categorías de cada una de las actividades que conforman a la Tercera Misión:

\section{1) Emprendimiento}

- Comercialización de Tecnologías

Con relación al registro de patentes se ha incrementado en un $120 \%$ por parte de las UPEs a partir del 2007, año en que el Programa Sectorial de Educación recomendaba a las IES de México realizar la protección de sus investigaciones. Sin embargo, aunque el incremento es significativo, existen UPEs que solo tienen tres patentes registradas durante este período de tiempo.

- Colaboración de investigadores en investigaciones no académicas:

En esta categoría la actividad que ha tenido un avance dentro de las UPEs es el número de organizaciones que colaboran en proyectos de investigación financiados por el Consejo Nacional de Ciencia y Tecnología, gracias a las diferentes convocatorias que son promovidas por dicho Consejo. El porcentaje de participación de organizaciones es del $33.45 \%$.

\section{2) Innovación}

- Investigación:
El número de académicos de las UPEs que participan en proyectos de investigación se ha visto incrementado en un 86.55\% desde el año 2007 a la actualidad debido a la contribución en proyectos de investigación con el Consejo Nacional de Ciencia y Tecnología y en menor escala con organizaciones gubernamentales y no gubernamentales; lo anterior, ha permitido que dichas instituciones obtengan financiamiento externo; sin embargo, no se cuenta con información acerca de los ingresos derivados de dichos contratos de investigación ni tampoco, con el número de contratos establecidos con organismos no académicos.

\section{3) Compromiso social:}

De acuerdo con la ANUIES (2016:79) "no existen datos estadísticos precisos acerca de las actividades que realizan las UPEs con relación al compromiso social ya que, para algunas de ellas, con realizar actividades de vinculación es suficiente para cumplir con su contribución al desarrollo social y económico".

No obstante, dicha asociación recomienda a sus integrantes incorporar actividades que impacten en los ámbitos social, económico y ambiental; además, menciona en el documento que como son instituciones públicas reguladas por disposiciones del gobierno, es necesario diseñar una política de Estado que permita la formulación de un proyecto de nación (ANUIES, 2016).

\section{Conclusiones}

La investigación documental exhibe que, de los 49 indicadores propuestos por Molas-Gallart et al., (2002), solo se obtuvo información de tres; no obstante, esto no significa que las UPEs no realicen actividades de emprendimiento, innovación y compromiso social que coadyuven al desarrollo de su Tercera Misión; sin embargo, es imperativo diseñar mecanismos que permitan dar seguimiento, evaluar y difundir ante la sociedad sus resultados obtenidos por dichas actividades.

Por otra parte, aunque las UPEs se encuentran en un proceso de adaptación con la vinculación con empresas, es necesario que se incluyan trabajos de investigación orientados a la generación y aplicación del conocimiento en problemas sociales ya que la finalidad de la Tercera Misión es desarrollar actividades donde se impulse el desarrollo económico y social de la región o país donde se encuentra la universidad.

Por lo tanto, para responder el objetivo de este trabajo de investigación, se llega a la conclusión que las UPEs están incorporando actividades de emprendimiento, innovación y compromiso social, dentro de sus planes de desarrollo; no obstante, se encuentran en un proceso de adaptación de dichas actividades, por lo tanto, actualmente se puede considerar que es poco significativo con la vinculación de la sociedad.

\section{Referencias}

UNESCO, (1998). Conferencia Mundial sobre la Educación Superior. La educación superior en el siglo XXI Visión y acción. Tomo Uno. Recuperado de: 
http://unesdoc.unesco.org/images/0011/001163/116345 s.pdf

Organización para la Cooperación y el Desarrollo Económico (2007). La Educación Superior y las Regiones: Globalmente Competitivas, Localmente Comprometidas, 1-198. Recuperado de: https://www.oecd.org/edu/imhe/laeducacionsuperioryla sregionesglobalmentecompetitivaslocalmentecomprom etidas.htm

Clark, B. (1998). Creating Entrepreneurial Universities: Organizational Pathways of Transformation. [Versión DX Reader]. Inglaterra: Oxford. Recuperado de http://www.finhed.org/media/files/05Clark_Creating_Entrepreneurial_Universities.pdf

Vilalta, J. (2013). La tercera misión universitaria. Innovación y transferencia de conocimientos en las universidades españolas. (F. E. Educación, Ed.). En Studia XXI. (4), 7-9. Recuperado de http://www.studiaxxi.com/site/wp-content/uploads/00.Cuaderno-de-trabajo-4-print.pdf Veblen, T. (1899). Teoría de la clase ociosa. 1-421 En elaleph.com. Recuperado de: http://argentina.indymedia.org/uploads/2012/10/teoria_ de_la_clase_ociosa.pdf

Associación Catalana d'Universitats Públiques (2008). Libro Blanco de la Universidad de Cataluña. Barcelona: Associació Catalana d'Universitats Públiques. Recuperado de: http://www.acup.cat/sites/default/files/libro-blanco.pdf

Galindo, J. Sanz, P., De Benito, J. (2011). La Universidad ante el reto de la Transferencia del Conocimiento 2.0: Análisis de las herramientas digitales a disposición del Gestor de Transferencia. En Investigaciones Europeas de Dirección y Economía de la Empresa. 17, (3), 111126. Recuperado de: www.elsevier.es/es-revistainvestigaciones-europeas-direccion-economia-empresa345

Bueno E. y Casiani F. (2007). La Tercera Misión de la Universidad Enfoques e Indicadores Básicos para su Evaluación. En Revista Economía Industrial, 366, 43 59. Recuperado

de: http://www.minetur.gob.es/Publicaciones/Publicacione speriodicas/EconomiaIndustrial/RevistaEconomiaIndus trial/366/43.pdf

Miranda, A., García, O. y Pérez, R. (2016). ¿Qué papel juegan actualmente las universidades públicas en la transferencia de conocimiento y la innovación tecnológica? El testimonio de profesoresinvestigadores de dos Universidades Públicas Estatales del país. En 5 Congreso Nacional de Ciencias Sociales. $\mathrm{La}$ ciencia emergente de las ciencias sociales. Conocimiento, Crítica e Intervención. 4-25. México: Centro Universitario de Ciencias Sociales y Humanidades de la Universidad de Guadalajara. Recuperado www.comecso.com/5congreso/EJE3.pdf

Asociación Nacional de Universidades e Instituciones de Educación Superior. (2016). Plan de Desarrollo Institucional. Visión 2030. México. Recuperado de: http://www.anuies.mx/media/docs/avisos/pdf/PlanDesa rrolloVision2030.pdf
Molas-Gallart, J., Salter, A., Patel, P., Scott, A. y Duran, X., (2002). Measuring Third Stream Activities. En: Science and Technology Policy Research. 1-48. Recuperado https://www.academia.edu/RegisterToDownload\#Dow nload

World Economic Forum (2016). The Global Competitiveness Report 2015-2016. Recuperado de: http://reports.weforum.org/global-competitivenessreport-2015-2016/competitiveness-rankings/

Asociación Nacional de Universidades e Instituciones de Educación Superior (2017a). En Anuario Estadístico de Educación Superior-Licenciatura. (Consultado el 10 de octubre de 2017).

Secretaria de Educación Pública. (2018). Principales Cifras del Sistema Educativo Nacional 2017-2018. Recuperado de: https://www.planeacion.sep.gob.mx/Doc/estadistica_e_ indicadores/principales_cifras/principales_cifras_2017 _2018_bolsillo.pdf

Universidad Nacional Autónoma de México (2017). Estudio Comparativo de las Universidades Mexicanas. En Explorador de Datos UNAM. (Consultado el 4 de diciembre de 2018)

Consejo Nacional de Ciencia y Tecnología (2017). Sistema Nacional de Investigadores. Recuperado de: https://datos.gob.mx/busca/dataset/sistema-nacionalde-investigadores

North, D. (1993). Instituciones, cambio institucional y desempeño económico. [Versión DX Reader]. Barcelona- México. Fondo de Cultura Económica. Recuperado de: https://dialnet.unirioja.es/descarga/articulo/4833969.pd $\mathrm{f}$

Coase, R. (1937). The Nature of the Firm. En Económica, New Series, 4. (16), 386-405. Recuperado de https://www.colorado.edu/ibs/es/alston/econ4504/readi ngs/The $\% 20$ Nature $\% 20$ of $\% 20$ the $\% 20$ Firm $\% 20$ by $\% 20$ Coase.pdf

Willamson, O. (1979). Transaction-Cost Economics: the Governance of Contractual Relation. En Journal of Law and Economics, 22 (2), 233-261. Recuperado de: https://www.business.illinois.edu/josephm/BA549_Fall $\% 202010 /$ Session\%203/Williamson\%20\%281979\%29. pdf

Ostrom E. (1990). Governing the Commons. [Versión DX Reader]. Cambridge University Press. Recuperado de: http://wtf.tw/ref/ostrom_1990.pdf

North, D. (1990). La Nueva Economía Institucional. En Revista Libertas, 12(142), 1-6. Recuperado de: http://www.eseade.edu.ar/wpcontent/uploads/2016/08/North.pdf

Searle, J.R. (2005). What is an Institution? En: Journal of Institutional Economic, 1, (1), 1-22. doi: http.//doi.org. /10.1017/S1744137405000020

Larrán-Jorge, J. y Andrades-Peña, F. (2015). Análisis de la responsabilidad social universitaria desde diferentes enfoques teóricos. En: Revista iberoamericana de educación superior, 6(15), 91-107. Recuperado de: http://www.scielo.org.mx/scielo.php?script=sci_arttext \& pid=S2007-28722015000100005\&lng=es\&tlng=es. 
Carrasco, I. y Castaño, M. S. (2012). La Nueva Economía Institucional. En Información Comercial EspañolaRevista de Economía, 865, 43-54. Recuperado de: http://www.revistasice.com/CachePDF/ICE_865_43_5 4_5BCB3F74AF28A440DCF8EF9FFFBA0496.pdf

Vaca, R., Moreno, M.J., Riquel, F. (2007). Análisis de la responsabilidad social corporativa desde tres enfoques: stakeholders, capital intelectual y teoría institucional. En Ayala (coord.), Conocimiento, innovación y emprendedores: camino al futuro. Universidad $\mathrm{La}$ Rioja, 3130-3143. Recuperado de: https://dialnet.unirioja.es/servlet/articulo?codigo=2234 8

Scott, R. (2013). Institutions and organizations: Ideas, interests, and identities. [Version DX Reader]. Saga Publications. Recuperado de: ttps://books.google.com.mx/books?hl=es\&lr=lang_es|la ng_en\&id=NbQgAQAAQBAJ\&oi=fnd\&pg=PP1\&dq= institutions+and+organizations+scott\&ots $=\mathrm{hFQdjJlk1F}$ $\&$ sig=rR40BRU8rbumZwhFqmEdUM5Wvls\#v=onepa ge\&q=institutions $\% 20$ and $\% 20$ organizations $\% 20$ scott $\&$ $\mathrm{f}=$ false

Powell W. y DiMaggio, P. (1999). El nuevo institucionalismo en el análisis organizacional, 104125. [Versión DX Reader]. México. Fondo de Cultura Económica. Recuperado de: http://www.inap.org.mx/portal/images/RAP/el\%20nuev o\%20intitucionalismo\%20en\%20el\%20analisis\%20org anizacional.pdf

Guzmán A. y Trujillo, M. (2008). Procesos de isomorfismo en la Facultad de Administración de la Universidad de los Andes. En Revista Universidad-Empresa, 7 (15), 219-236. Recuperado de: http://www.redalyc.org/articulo.oa?id=187214457011

De la Torre, E., Casiani, F. y Sagarra M. (2018). Defining typologies of universities through a DEA-MDS analysis: An institutional characterization for formative evaluation purposes. En Research Evaluation, 1-16. doi: $10.1093 /$ reseval/rvy024 